\title{
Field emission mitigation studies in the SLAC Linac Coherent Light Source II superconducting rf cavities via in situ plasma processing
}

\author{
Bianca Giaccone®* \\ Fermi National Accelerator Laboratory, Kirk Road and Pine Street, Batavia, Illinois 60510, USA \\ and Physics Department, Illinois Institute of Technology, \\ 10 West 35th Street, Chicago, Illinois 60616, USA \\ Martina Martinello, Paolo Berrutti®, Oleksandr Melnychuk@, \\ Dmitri A. Sergatskov, and Anna Grassellino \\ Fermi National Accelerator Laboratory, Kirk Road and Pine Street, Batavia, Illinois 60510, USA \\ Dan Gonnella and Marc Ross (1) \\ SLAC National Accelerator Laboratory, 2575 Sand Hill Road, Menlo Park, California 94025, USA \\ Marc Doleans \\ Oak Ridge National Laboratory, 1 Bethel Valley Road, Oak Ridge, Tennessee 37830, USA \\ John F. Zasadzinski \\ Physics Department, Illinois Institute of Technology, 10 West 35th Street, Chicago, Illinois 60616, USA
}

(Received 16 September 2020; accepted 25 January 2021; published 19 February 2021)

\begin{abstract}
Field emission is one of the main factors that can limit the performance of superconducting radio frequency cavities. To reduce possible field emission in the Linac Coherent Light Source II (LCLS-II), we are developing plasma processing for $1.3 \mathrm{GHz}$ nine-cell cavities. The ultimate goal of plasma processing will be to apply the technique in situ in the cryomodules in order to mitigate hydrocarbon-related field emission without disassembling them. Herein is presented the first systematic study of plasma processing applied to LCLS-II superconducting radio frequency cavities. Having developed a new method of plasma ignition for LCLS-II cavities, we applied plasma processing to $1.3 \mathrm{GHz}$ cavities starting with a clean nitrogen doped cavity and proceeding with studying natural field emission and artificially contaminated cavities. All the cavities were cold tested before and after plasma cleaning in order to compare their performances. It was proved that this technique successfully removes carbon-based contamination from the cavity iris and that it is able to eliminate field emission in a naturally field emitting cavity. The effect of plasma processing on cavities exposed to vacuum failures was also investigated, showing positive results in some cases. This work shows how successful plasma processing is in removing hydrocarbon related contamination from the cavity surface without affecting the high Q-factors and quench fields characteristic of nitrogen doped cavities.
\end{abstract}

DOI: 10.1103/PhysRevAccelBeams.24.022002

\section{INTRODUCTION}

A collaboration among Fermi National Accelerator Laboratory (FNAL), SLAC National Accelerator Laboratory and Oak Ridge National Laboratory (ORNL) is working to develop plasma processing for LCLS-II [1,2]

\footnotetext{
*giaccone@fnal.gov
}

Published by the American Physical Society under the terms of the Creative Commons Attribution 4.0 International license. Further distribution of this work must maintain attribution to the author(s) and the published article's title, journal citation, and DOI.
1.3 GHz nitrogen doped [3-6] cavities. Linac Coherent Light Source II (LCLS-II) is the LCLS X-ray Free Electron Laser (XFEL) [7,8] upgrade, and will utilize a superconducting linear accelerator, along with other cuttingedge components, to produce an x-ray laser beam $10^{4}$ times brighter than LCLS $[9,10]$.

The scope of plasma processing is to be applied in situ in LCLS-II cryomodules to help mitigate hydrocarbon-related field emission in nine-cell cavities.

Field emission (FE) is a phenomenon that limits the accelerating gradient at which a cavity can operate [11]; it consists of electron emission from regions of the cavity surface with intense applied electric field [12]. The emitted 
electrons are accelerated by the electric field and impact the cavity walls depositing heat and creating bremsstrahlung $x$ rays. These electrons can also interact with and disrupt the beam passing through the cavity. The $\mathrm{x}$ rays produced by the FE can cause radiation damage to the cryomodule's components, decreasing operational lifetime. Once the FE is activated, it limits the cavity's accelerating field and causes a degradation in quality factor due to the additional dissipation introduced by the emitted electrons. If $\mathrm{FE}$ is severe, it can cause thermal breakdown of the cavity and can also activate the beam line, causing induced radioactivity in the cavity.

Sources of FE are contaminants (dust or metal particles) or cavity surface defects that cause local enhancement of the FE current. In addition, the presence of even a few monolayers of hydrocarbons, or other adsorbate gases, on the cavity surface can further decrease the $\mathrm{Nb}$ work function [13], facilitating FE. The origin of hydrocarbon contamination on the cavity inner surface is not completely understood; however, its presence has been reported in the literature in multiple cases [14-16] and carbon has been observed in both adventitious form and as local contamination on the $\mathrm{Nb}$ surface. Doleans et al. report in [14] that evidence of volatile hydrocarbon has been found through residual gas analysis on thermally cycled Spallation Neutron Source (SNS) [17]) cryomodules; they explain that these signals must originate from the released gases that were previously condensed on the cavity walls at cryogenic temperature or from species produced during accelerator operation by the interaction of electrons with the cavity surface contaminants.

Plasma processing can be used on superconducting radio frequency (SRF) cavities in situ in the cryomodules to remove the hydrocarbon contamination and restore the niobium work function obtaining a decrease in FE and a corresponding increase in the accelerating gradient. This technique was first applied to SRF cavities at ORNL, where Doleans et al. developed plasma cleaning for SNS high beta $805 \mathrm{MHz}$ cavities [18]. Plasma processing has been applied to multiple SNS cryomodules, both off-line and on-line, showing improvement in the accelerating gradient [19]. Starting from SNS experience, a new method of plasma ignition for LCLS-II 1.3 GHz TESLA-shaped [20] cavities has been developed by Berrutti et al. at FNAL [21-23]. Studies on plasma ignition are also being conducted at the Institute of Modern Physics, Chinese Academy of Sciences, on half wave resonators (HWRs). Wu et al. used an experimental setup that replicates the cavity assembled in the cryomodule to study plasma ignition with the $\mathrm{Ar}-\mathrm{O}_{2}$ gas mixture in HWR cavities [24] and studied the effect of plasma processing on an HWR cavity contaminated with methane gas [25]. Huang et al. [26] applied helium and plasma processing to low-beta HWR.

In this paper we present the results of plasma processing applied to multiple $1.3 \mathrm{GHz}$ cavities. The cavities were cold tested before and after plasma processing in order to compare their performances in terms of quality factor $\left(\mathrm{Q}_{0}\right)$ and radiation versus accelerating field $\left(\mathrm{E}_{\mathrm{acc}}\right)$. An $\mathrm{N}$-doped [3] single-cell was used for the first plasma processing test in order to study the possible effects of the plasma on the surface treatment. Afterwards, plasma cleaning was applied on two nine-cell cavities with natural FE (meaning with contamination introduced during the nominally clean assembly process and, therefore with FE of unknown source, not caused by intentional contamination introduced in the cavity). Given the results of these tests, it was decided to investigate the efficacy of plasma processing on cavities artificially contaminated with carbon-related (C-related) sources or through vacuum failure simulations.

\section{EXPERIMENTAL SYSTEM AND PLASMA PARAMETERS}

Plasma cleaning uses a glow discharge [27,28] ignited inside the cavity volume to remove the hydrocarbons from the niobium surface, restoring the $\mathrm{Nb}$ work function and causing a decrease in FE [29]. An inert gas (neon) is injected into the cavity to ignite and sustain the plasma and a small percentage of $\mathrm{O}_{2}$ is added to the $\mathrm{Ne}$. The oxygen molecules are dissociated in the plasma and the reactive oxygen binds with the hydrocarbons on the surface, creating volatile by-products that are easily pumped out of the cavity.

The glow discharge is ignited inside rf volume, cell by cell, using the cavity's resonant modes. The scope of plasma processing is to be used in situ in the cryomodule, so this technique relies only on the hardware present in the cryomodule cavity's assembly. The SNS method for plasma ignition is dual tone excitation [14], which uses the fundamental passband. For LCLS-II $1.3 \mathrm{GHz}$ cavities, it is not possible to ignite the glow discharge using the fundamental power coupler (FPC). As Berrutti et al. explain in [21], the cavity quality factor $\mathrm{Q}_{0}$ and the FPC $\mathrm{Q}_{\text {ext }}$ are highly mismatched at room temperature [30]. Therefore, the approach taken here for LCLS-II cavities is a new plasma ignition method, developed by Berrutti et al. using the higher order modes (HOMs) and the HOM couplers [22]. Modes belonging to two dipole passbands are used to ignite the glow discharge in the central cell and to plasma process the entire cavity. The procedure is composed of two identical rounds: during each round, all the cavity cells are plasma processed. Using the newly developed HOM ignition method, the glow discharge is ignited in the central cell and then immediately transferred to cell number 9, passing through adjacent cells. Once arrived in the desired cell, the rf driving frequency is tuned in order to increase the resonant peak's frequency shift and to maximize the plasma density $[22,31,32]$. The cell is processed for $50 \mathrm{~min}$, then the plasma density is decreased and the glow discharge is transferred to the adjacent cell using a combination of resonant dipole modes. The 


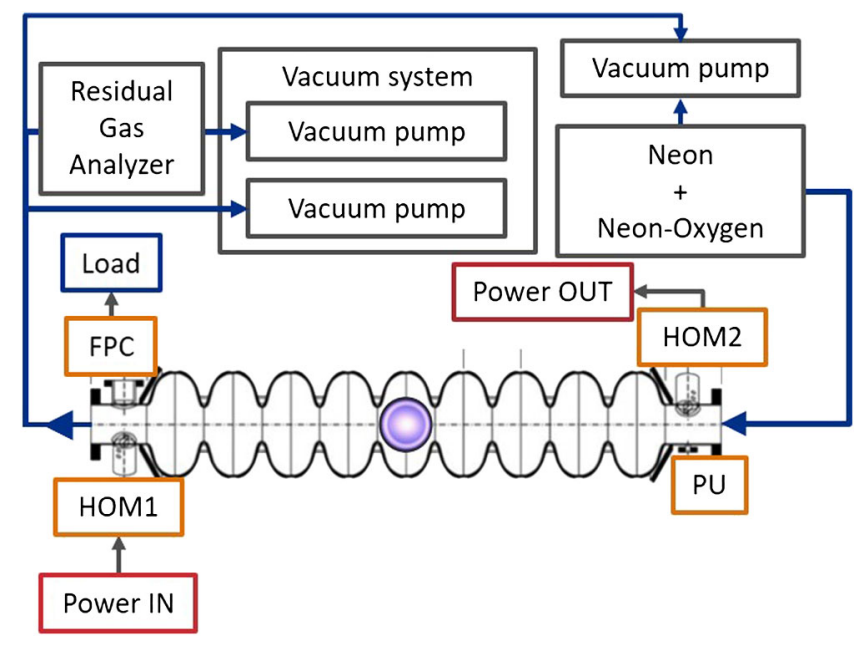

FIG. 1. Block scheme of the experimental setup used for plasma processing. The cavity is placed inside a portable cleanroom, and all the connections between the cavity and the gas/ vacuum system take place inside the cleanroom.

procedure is repeated until all the cavity cells, from \#9 to $\# 1$, have been plasma processed. Once the first round is completed, the second identical round is performed.

Each cell is processed for a total of $100 \mathrm{~min}$. The duration of each round has been decided using the data collected by the residual gas analyzer (RGA) assembled on the pumping system: it has been observed that peaks in the C-related signals are often present when the plasma is ignited in (or transferred to) a new cell. These peaks usually decrease to the background level in approximately $30 \mathrm{~min}$ or less. During the second round of plasma processing, there is usually no increase in the C-related signals. An example of the partial pressure signals registered by the RGA is later shown in Fig. 7. The RGA is used during the entire plasma cleaning procedure to monitor the concentration of oxygen in the mixture and the by-products of the reaction between $\mathrm{O}_{2}$ and the hydrocarbons on the cavity surface.

Plasma processing on LCLS-II cavities is performed at room temperature, using a mixture of neon with approximately $1 \%-1.5 \%$ oxygen, for a total gas pressure of approximately 50-100 mTorr; a block scheme of the

TABLE I. Summary of the parameters currently used for plasma processing. The first row pertains to the ignition of the plasma in the central cell, the second row to the parameters used to process each cell. $\mathrm{P}_{\mathrm{FWD}}$ indicates the total power forwarded to the cavity; $\Delta \mathrm{f}$ is the frequency shift of the resonant peak caused by the ignition of the plasma and the tuning of the rf driving frequency to maximize the plasma density.

\begin{tabular}{lcccc}
\hline \hline Frequency range & $\mathrm{P}_{\mathrm{FWD}}$ & Pressure & $\mathrm{O}_{2}$ & $\Delta \mathrm{f}$ \\
\hline$(\mathrm{MHz})$ & $(\mathrm{W})$ & $(\mathrm{mTorr})$ & $(\%)$ & $(\mathrm{MHz})$ \\
\hline $1825-1835$ & $75-90$ & $50-100$ & $1-1.5$ & $0.5-1.5$ \\
$1600-1750$ & 10 & $50-100$ & $1-1.5$ & $10-15$ \\
\hline \hline
\end{tabular}

experimental setup in use at FNAL is shown in Fig. 1, and a detailed schematic of the experiment and a description of the method used to ignite and transfer the plasma across the cavity are contained in [22]. The set of parameters currently used (summarized in Table I) has been developed during the first plasma processing experiment on a nine-cell cavity: however, it has not yet been optimized. Studies to identify the set of parameters (pressure, duration, oxygen percentage, plasma density/frequency shift) that maximizes the plasma efficiency are currently ongoing.

\section{A. Removal study of C-based contamination from cavity iris}

After the plasma ignition studies on $1.3 \mathrm{GHz}$ cavities [22], the first plasma processing test was performed on a nine-cell cavity assembled with viewports on the beam tubes. A permanent marker was used to introduce C-based contamination on the iris of one end cell. Permanent marker ink is composed of hydrocarbon chains, as shown by the energy dispersive $x$-ray spectroscopy (EDS) analysis in Fig. 2, and it was previously used for plasma processing studies at SNS [29,31]. Two permanent markers (black and red ink) were used to draw eight dots on the cavity iris. We applied plasma processing to the contaminated cell for 19 h: Fig. 3 shows the initial and final state of the cavity, while Fig. 4 offers a close-up view of the initial, intermediate and final states.
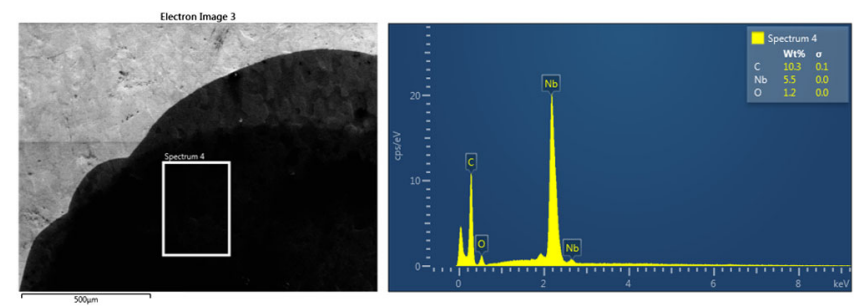

FIG. 2. Permanent marker ink analyzed with scanning electron microscopy (SEM) on the left and energy dispersive $\mathrm{x}$-ray spectroscopy (EDS) on the right. A $\mathrm{Nb}$ sample is used as substrate.

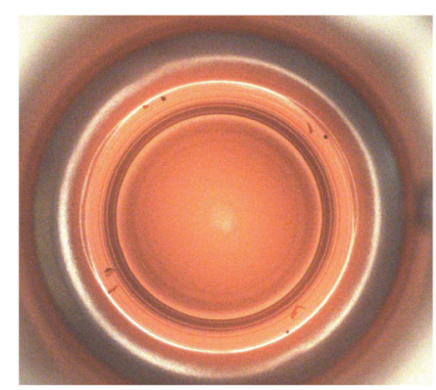

(a)

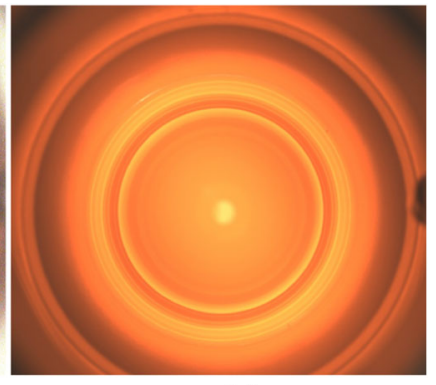

(b)
FIG. 3. Glow discharge ignited in the end cell contaminated with permanent marker dots: (a) initial state, (b) final state after 19 hours of plasma processing. 


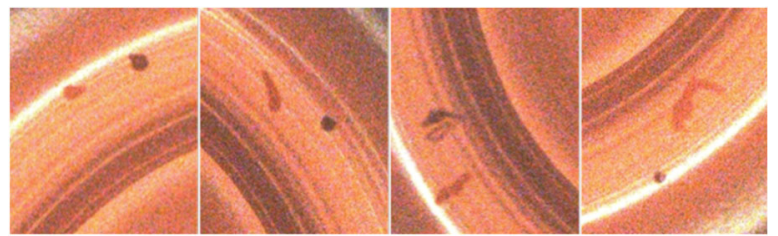

(a)

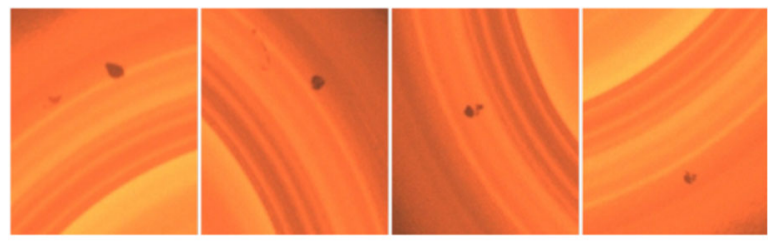

(b)

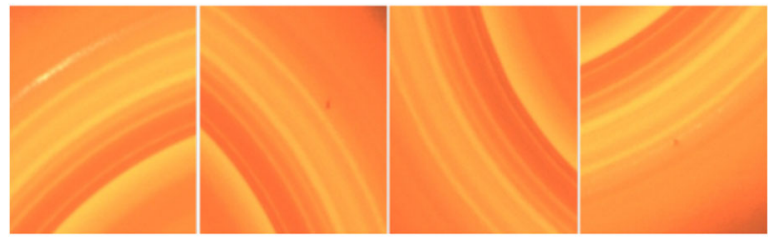

(c)

FIG. 4. Close-up view of the contamination showed in Fig. 3. The initial state of the contamination is shown in (a), with the final state in (c); (b) shows the progress after 5 hours of plasma processing.

With this test, we confirmed that plasma processing is able to remove C-based contamination from the cavity iris, the area most problematic for FE. The experiment has also allowed us to develop a first plasma recipe in terms of duration of the process, $\mathrm{O}_{2}$ percentage, pressure, and plasma density. This set of parameters, summarized in Table I, was later used to apply plasma processing on multiple LCLS-II cavities, both single-cell and nine-cell cavities.

\section{PLASMA PROCESSING AND RF TESTS ON 1.3 GHZ CAVITIES}

Plasma processing was applied to LCLS-II cavities, both single-cell and nine-cells, and the effectiveness of plasma cleaning was measured in terms of $\mathrm{Q}_{0}$ versus $\mathrm{E}_{\mathrm{acc}}$ and radiation versus $\mathrm{E}_{\text {acc }}$ curves. All the cavities were cold tested before and after plasma processing in order to compare their performances.

The first test was carried out on a clean nitrogen doped cavity, with the following two conducted on naturally field emitting nine-cell $\mathrm{N}$-doped cavities. The subsequent studies were conducted on artificially contaminated cavities in order to investigate the efficacy of plasma processing under different circumstances. Table II summarizes the tests conducted on LCLS-II $1.3 \mathrm{GHz}$ cavities.

All the rf cold tests were done in the vertical test stand (VTS) facility at FNAL, following the measurement method explained in [33]. The cryogenic Dewars are equipped with two radiation detectors [34] positioned on the top and on the bottom of the Dewar where the cavity is placed for the cold test. To reliably compare the results of the rf tests performed before and after plasma processing, we attempted to always test each cavity in the same VTS Dewar and, when possible, in the same position inside the Dewar. In some cases it was not possible to test the cavity in the same Dewar: this is indicated in the text.

All the plots in this paper use the following symbols: solid symbols for $\mathrm{Q}_{0}$ versus $\mathrm{E}_{\text {acc }}$ curves, empty and halffilled symbols for the radiation versus $\mathrm{E}_{\mathrm{acc}}$ curves, with empty symbols used for the radiation detector located on top of the cryogenic Dewar and vertically half-filled symbols for the bottom radiation detector.

\section{A. Baseline test on N-doped cavity}

$\mathrm{N}$-doping is a surface treatment developed at FNAL that has allowed increase of the cavity $\mathrm{Q}_{0}$ by a factor of $3[3,5]$. The recipe used to N-dope LCLS-II cavities is called " $2 / 6$," which consists of baking the cavity in vacuum ( $p<1 \times 10^{-6}$ Torr) at $800^{\circ} \mathrm{C}$ : once the temperature is stable nitrogen is injected into the furnace at a pressure of $25 \mathrm{mTorr}$ for $2 \mathrm{~min}$. Afterwards the vacuum is restored and the cavity undergoes 6 min of annealing. After the doping, $5 \mu \mathrm{m}$ are removed from the inner cavity surface through electropolishing (EP) in order to eliminate possible nitrides. After $5 \mu \mathrm{m}$ of EP, only the interstitial nitrogen remains in

TABLE II. Summary of the $1.3 \mathrm{GHz}$ single-cell and nine-cell TESLA-shaped cavities cold tested for plasma processing studies. The terminology " $\mathrm{n} / \mathrm{m}$ " for the nitrogen doping is to be interpreted as $n$ minutes in 25 mTorr of nitrogen and $m$ minutes of annealing in vacuum.

\begin{tabular}{llll}
\hline \hline Cavity & Surface treatment & & Contamination \\
\hline Single-cell & "2/6" N-doping & & Test scope \\
Nine-cell & "3/60" N-doping & Natural FE & Plasma processing effect on N-doping \\
Nine-cell & "3/60" N-doping & Natural FE & Removal of natural FE \\
Single-cell & '2/6" N-doping & Aquadag® & Removal of C-contamination \\
Nine-cell & EP & Vacuum failure simulated inside cleanroom & FE mitigation \\
Single-cell & "2/6" N-doping & Vacuum failure simulated outside cleanroom & FE mitigation \\
Nine-cell & "2/6" N-doping & Vacuum failure simulated outside cleanroom & FE mitigation \\
Nine-cell & "2/6" N-doping & Vacuum failure simulated outside cleanroom & FE mitigation \\
\hline \hline
\end{tabular}




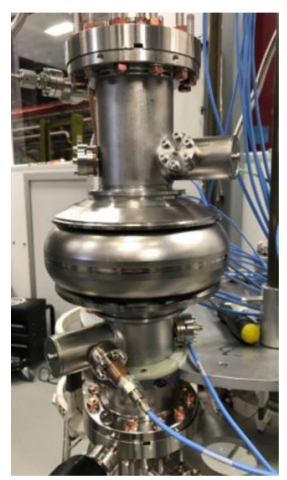

(a)

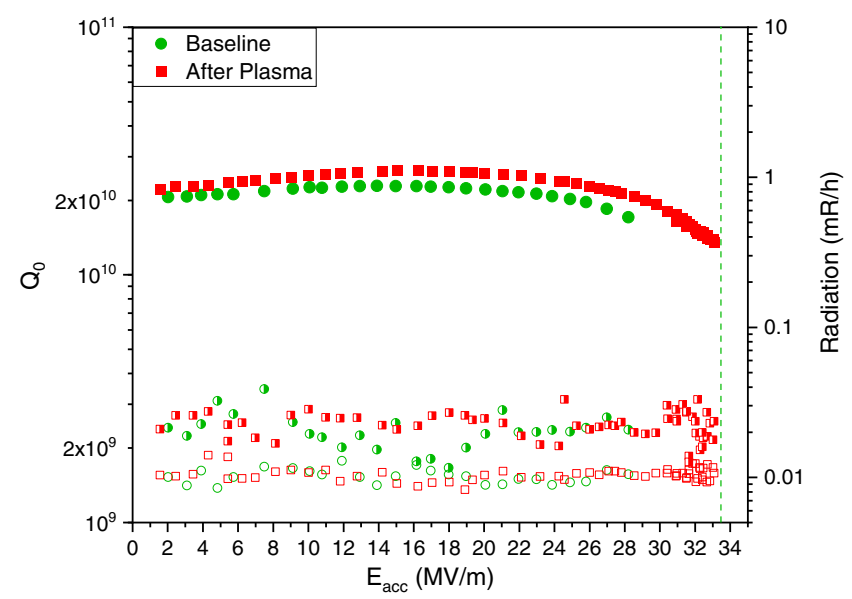

(b)

FIG. 5. Part (a) shows a single-cell cavity with HOM couplers. Part (b) shows the results of the rf tests measured at $2 \mathrm{~K}$ before (green) and after (red) plasma processing. The baseline rf test has been intentionally limited at $28 \mathrm{MV} / \mathrm{m}$ at $2 \mathrm{~K}$ to avoid quenching. The quench field was reached at $\mathrm{E}_{\mathrm{acc}}=33.5 \mathrm{MV} / \mathrm{m}$ during the $1.4 \mathrm{~K}$ baseline test and the vertical dotted line indicates the quench field value. As explained in Sec. III, solid symbols are used to plot the $\mathrm{Q}_{0}$ vs $\mathrm{E}_{\text {acc }}$ curve, empty symbols for the radiation detected from the top detector, and half-filled symbols for the bottom radiation detector.

the niobium with a concentration on the order of $100 \mathrm{ppm}$. $\mathrm{N}_{2}$ atoms are absorbed as interstitial impurities in the niobium lattice and cause a reduction of the Mattis-Bardeen surface resistance $R_{B C S}$ with the accelerating field [35].

Since all $1.3 \mathrm{GHz}$ LCLS-II cavities are nitrogen doped $[4,6]$, we have started our studies by applying plasma cleaning to an $\mathrm{N}$-doped single-cell cavity in order to understand if this processing can affect the surface treatment.

The single-cell used for the test was built by welding together two end cells of a nine-cell cavity. The result is a single-cell cavity with HOM couplers on the beam tubes [see panel (a) in Fig. 5]. This characteristic makes it suitable for plasma processing, as it allows ignition of the glow discharge using the HOMs and, with only one cell to process, drastically reduces the duration of the cleaning.
The results of the rf cold tests obtained on the single-cell before and after plasma processing are shown in panel (b) of Fig. 5. We intentionally stopped the baseline test before quenching in order to measure the cavity $\mathrm{Q}_{0}$ at $1.4 \mathrm{~K}$, where it reached a quench field equal to $33.5 \mathrm{MV} / \mathrm{m}$. After the first vertical test, the cavity was connected to the vacuum/ gas and rf system used for plasma cleaning and processed for $16 \mathrm{~h}$ with $\mathrm{Ne}-\mathrm{O}_{2}$ plasma.

Comparing the rf tests measured before and after plasma processing, it is clear that plasma cleaning does not negatively affect the performance of the nitrogen doped single-cell; on the contrary, it preserves the high quality factor and quench field characteristics of $\mathrm{N}$-doped cavities.

\section{B. Naturally field emitting $\mathbf{N}$-doped cavities}

We used two cavities with natural FE to test the efficacy of plasma processing on FE of unknown source, i.e., emission not caused by artificial contamination but by contamination introduced during the nominally clean assembly process. The two nine-cell cavities exhibited $\mathrm{x}$ rays during the first vertical tests performed at FNAL. Both cavities were assembled with a second valve to allow the flow of gas for plasma processing and $\mathrm{rf}$ tested again after valve assembly. Afterwards, the two cavities were plasma processed and cold tested.

The top plot in Fig. 6 shows the results of the cold tests of the first nine-cell cavity; the curves registered before plasma processing show that the cavity quenched at $\mathrm{E}_{\mathrm{acc}}=$ $18.5 \mathrm{MV} / \mathrm{m}$ with $\mathrm{x}$-ray onset at $16 \mathrm{MV} / \mathrm{m}$. After plasma processing, the cavity reached $\mathrm{E}_{\text {acc }}=18 \mathrm{MV} / \mathrm{m}$, exhibiting no $\mathrm{x}$ rays. In this case, plasma processing completely removed FE. The fact that no change in quench field was observed suggests a hard quench, which was not due to FE.

The bottom plot in Fig. 6 shows the performance of the second cavity: the radiation onset before plasma processing was registered at $7 \mathrm{MV} / \mathrm{m}$; the test was stopped before the quench field due to intense radiation levels $\left(1.1 \times 10^{4} \mathrm{mR} / \mathrm{h}\right.$ at $\left.16.5 \mathrm{MV} / \mathrm{m}\right)$, and the final FE onset was measured at $7.8 \mathrm{MV} / \mathrm{m}$. The cold test conducted after plasma processing showed that the $\mathrm{x}$-ray onset decreased to $7 \mathrm{MV} / \mathrm{m}$ and the quality factor degraded. Also in this case, the rf test was interrupted due to intense FE. The $\mathrm{Q}_{0}$ degradation could be due to a higher ambient magnetic field during cooldown, which results in increased trapped magnetic flux in the cavity. The two rf tests measured on this cavity were carried out in different cryogenic Dewars, which could also explain the difference in trapped flux.

The fact that plasma processing was effective on one cavity with natural FE, but not on the second, indicates that the FE may originate from different sources. The evidence suggests that in the first cavity the FE was caused by hydrocarbon contamination and that plasma processing was effective in removing it thanks to the reactive oxygen, present in the glow discharge, that binds with the $\mathrm{H}_{\mathrm{x}} \mathrm{C}_{\mathrm{y}}$ and creates volatile by-products. In the second cavity, the FE 


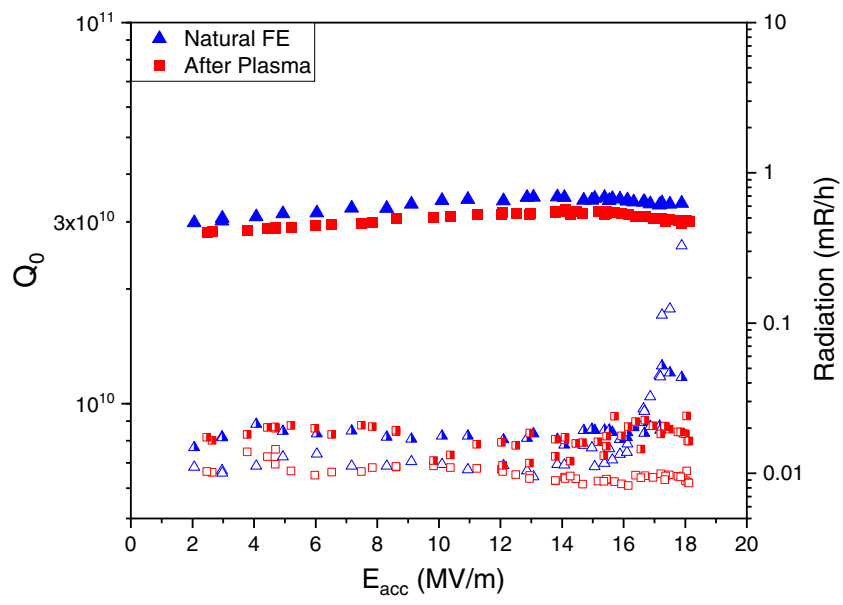

(a)

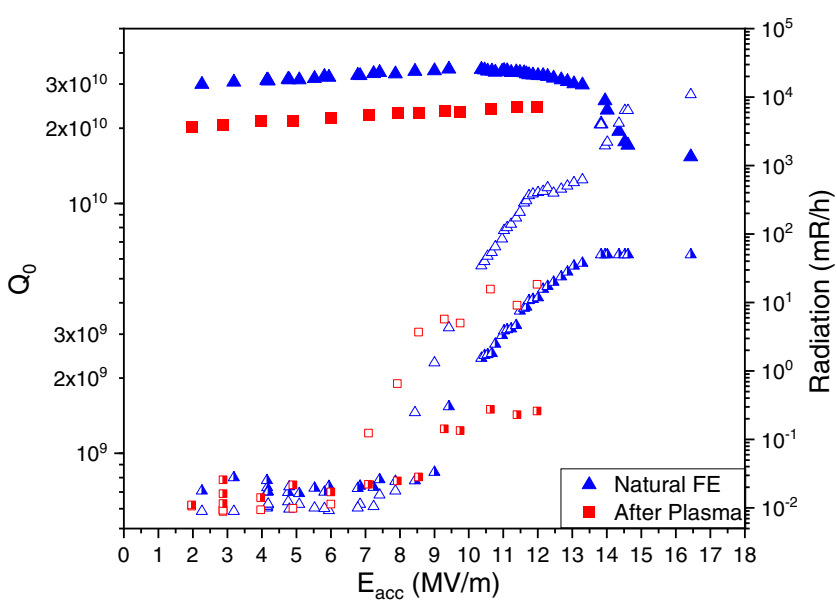

(b)

FIG. 6. Part (a) shows the results of the first naturally field emitting cavity: in blue is $\mathrm{Q}_{0}$ and radiation level vs $\mathrm{E}_{\mathrm{acc}}$ performed before plasma processing. The $\mathrm{x}$-ray onset is $16 \mathrm{MV} / \mathrm{m}$ before plasma processing; after plasma processing the FE was completely removed: the rf test measured after the treatment shows no $\mathrm{x}$ rays. Part (b) shows the rf tests of the second cavity. The test was stopped before quenching due to intense $\mathrm{x}$ rays. The red curves show the performance after plasma processing: no increase in performance has been registered after the cleaning. Solid symbols are used to plot the $\mathrm{Q}_{0}$ vs $\mathrm{E}_{\text {acc }}$ curve, empty symbols for the radiation detected from the top detector, and half-filled symbols for the bottom radiation detector.

may instead not be caused by hydrocarbons, but it could be due to surface defects or metal flakes on the cavity surface. If that is the case, it is expected that the plasma cleaning would exert little effect in mitigating FE since no volatile by-products are generated in the reaction.

\section{Residual gas analyzer}

A residual gas analyzer is used to monitor the composition of the gas pumped out of the cavities for the duration of the entire plasma cleaning. Figure 7 shows the RGA data

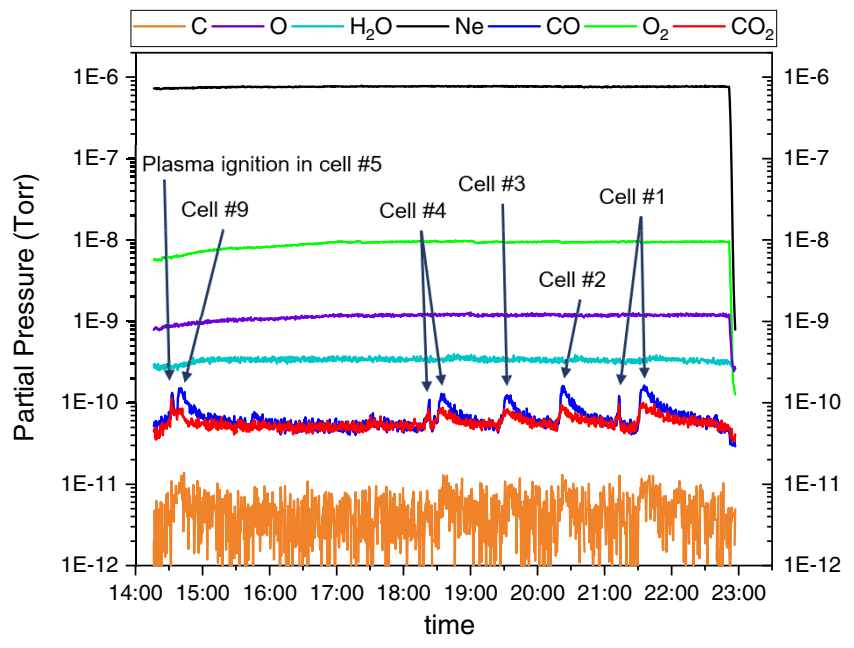

FIG. 7. Example of RGA plot acquired during the first day of plasma processing of a nine-cell cavity. Cell \#4 and cell \#1 exhibit a double peak due to the plasma being accidentally turned off while reaching the desired plasma density.

acquired during the first round of plasma processing applied to the first naturally field emitting cavity, which represents a typical example of the RGA data registered during plasma processing. During the first round of plasma cleaning, the RGA data often show $\mathrm{C}, \mathrm{CO}$ and $\mathrm{CO}_{2}$ peaks corresponding with the moment when the plasma is ignited in, or transferred to, a new cell. The increase in the $\mathrm{C}$-related signals shows that the oxygen is reacting with the $\mathrm{H}_{\mathrm{x}} \mathrm{C}_{\mathrm{y}}$ on the cavity surface.

The RGA can record masses from 1 to $300 u$; however, only the elements of interest are shown in this plot. In Fig. 7 the C-related peaks are clearly visible in correspondence with the plasma being initially ignited in cell \#5, then transferred to, and tuned, in cell \#9, and transferred and tuned in cell \#4, up to cell \#1. Cells from \#8 to \#5 do not exhibit prominent peaks in the C-related signals. This could be because, at the beginning of the procedure, the glow discharge is ignited in the central cell (first peak on the left in Fig. 7) and then moved from cell \#5 up to cell \#9, passing through all intermediate cells.

\section{C-based contamination}

We contaminated the single-cell cavity, previously used for the N-doping test, using a C-based paint in order to study the effectiveness of plasma processing on an artificial carbon contamination. Aquadag ${ }^{\circledR}$ [36], a conductive paint made of graphite and ultrapure water, was used to contaminate the cavity. A small drop of highly diluted paint was deposited on the cavity iris. Figure 8 shows images of pure and diluted Aquadag acquired with the scanning electron microscope (SEM). The Aquadag used to contaminate the single-cell iris was diluted by a factor $2 \times 10^{4}$, where the dilution was calculated as the ratio between the $\mathrm{H}_{2} \mathrm{O}$ and the Aquadag mass. 


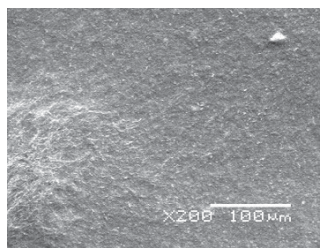

(a)

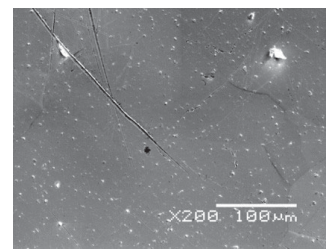

(b)

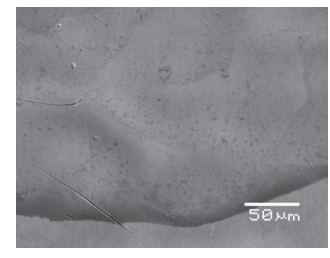

(c)

FIG. 8. Scanning electron microscope images of pure (a) and diluted Aquadag ${ }^{\circledR}$ on $\mathrm{Nb}$ substrate. The dilution factor has been calculated as the ratio of $\mathrm{H}_{2} \mathrm{O}$ mass to Aquadag mass. Parts (b) and (c) respectively show Aquadag diluted by factors of 100 and $2 \times 10^{4}$.

For this study it was not possible to cold test the cavity in the same cryogenic Dewar before and after plasma processing.

Figure 9 shows the results of the rf tests. The contaminated single-cell was plasma processed for $17 \mathrm{~h}$ between the two cold tests. In blue is the curve measured on the contaminated cavity; in comparison with the baseline test (here, the same curve shown in red in Fig. 5, is presented in green), it can be observed that the cavity exhibits

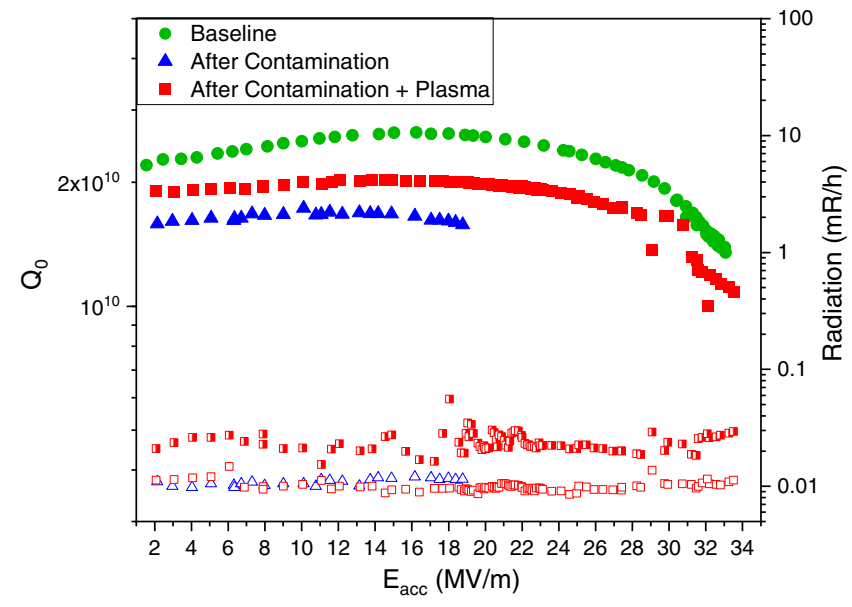

FIG. 9. Quality factor versus accelerating field curves measured on the contaminated cavity and after plasma cleaning. The contaminated cavity (blue triangle) exhibits a degradation in quench field and $\mathrm{Q}_{0}$. After plasma processing it exhibits an increase in quality factor $\left(\mathrm{Q}_{0}=2 \times 10^{10}\right.$ at $\left.16.4 \mathrm{MV} / \mathrm{m}\right)$ and a complete recovery in $\mathrm{E}_{\text {acc }}$ (the quench field is $33.5 \mathrm{MV} / \mathrm{m}$ ). In green is shown the baseline performance of the cavity before contamination. Solid symbols are used to plot the $\mathrm{Q}_{0}$ vs $\mathrm{E}_{\mathrm{acc}}$ curve, empty symbols for the radiation detected from the top detector, and half-filled symbols for the bottom radiation detector. degradation in the quality factor and quench field: $\mathrm{Q}_{0}=$ $1.7 \times 10^{10}$ at $\mathrm{E}_{\mathrm{acc}}=16.2 \mathrm{MV} / \mathrm{m}$, and the quench field is registered at $18.8 \mathrm{MV} / \mathrm{m}$. The radiation detector positioned at the bottom of the cryogenic Dewar was not functioning correctly during this cold test: however, the top radiation detector was connected, and no $\mathrm{x}$ rays were registered. After $17 \mathrm{~h}$ of plasma processing, the cavity exhibits an increase in quality factor $\left(\mathrm{Q}_{0}=2 \times 10^{10}\right.$ at $\left.\mathrm{E}_{\mathrm{acc}}=16.4 \mathrm{MV} / \mathrm{m}\right)$. Plasma processing increased the quench field by almost $15 \mathrm{MV} / \mathrm{m}$, restoring the initial quench field at $\mathrm{E}_{\mathrm{acc}}=33.5 \mathrm{MV} / \mathrm{m}$.

\section{Vacuum failure experiments}

A possible cause of cavity contamination is a vacuum leak or a complete vacuum loss. Multiple experiments were conducted on cavities exposed to air in order to understand whether plasma processing can be effective in mitigating $\mathrm{FE}$ in these scenarios. The tests were carried out under different conditions on both nine-cell and single-cell cavities. We refer to these tests as vacuum failure experiments (or simulations).

\section{Vacuum failure experiment inside the cleanroom}

We conducted the first test inside a cleanroom environment in order to introduce a controlled particulate amount. High pressure rinsing was used to clean the cavity and, after drying, it was slowly evacuated to high vacuum. To simulate the vacuum failure, the mini right angle valve (RAV) was opened while the cavity was in a class 10 cleanroom. The cavity quickly reached atmospheric pressure and, after exposure to this pressure for a few minutes, it was slowly evacuated to reach a pressure in the low $10^{-6}$. high $10^{-7}$ Torr range.

Plasma processing was applied twice to this cavity, each time using the standard parameters and duration (approximately $1 \mathrm{~h} 40 \mathrm{~min}$ per cell). After each complete plasma processing round, the cavity was rf tested at $2 \mathrm{~K}$. The $\mathrm{rf}$ tests on the contaminated cavity and after the second plasma processing were conducted in the same cryogenic Dewar, while the cold test after the first plasma cleaning was carried out in a different Dewar.

Panel (a) of Fig. 10 summarizes the performance of the cavity during the cold tests. In blue are the curves registered before plasma processing (on the contaminated cavity): the nine-cell reached a first quench at $7.5 \mathrm{MV} / \mathrm{m}$, and it was then possible to increase the power and measure the $\mathrm{Q}_{0}$ versus $E_{\text {acc }}$ curve up to $23 \mathrm{MV} / \mathrm{m}$ (with intermediate quenches at 20.5 and $22 \mathrm{MV} / \mathrm{m}$ ), when the cavity reached the final quench. The $\mathrm{x}$-ray onset was registered at $18.5 \mathrm{MV} / \mathrm{m}$ for the bottom radiation detector, and at $20 \mathrm{MV} / \mathrm{m}$ for the top detector. The cavity was also tested at $1.4 \mathrm{~K}$, and the purple curves show the radiation level registered during the final power rise (intentionally stopped before quench): the maximum radiation level switched 


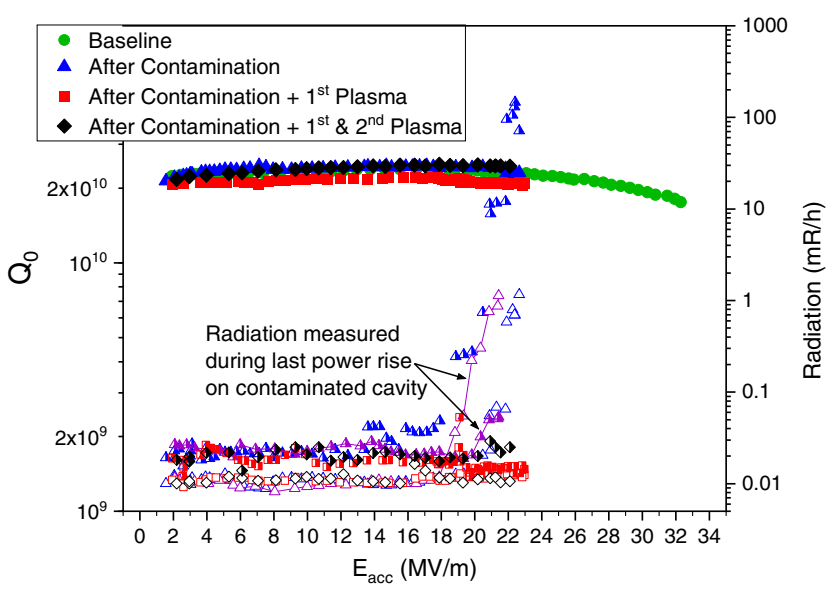

(a)

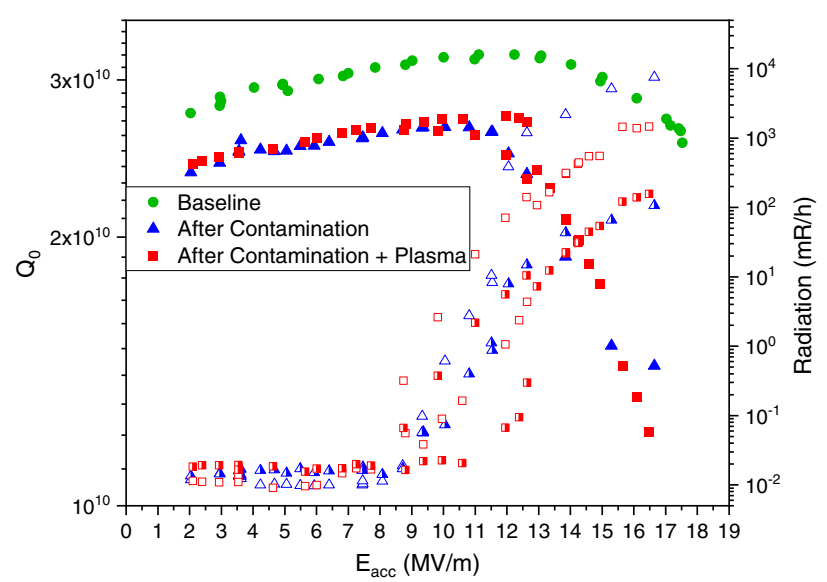

(c)

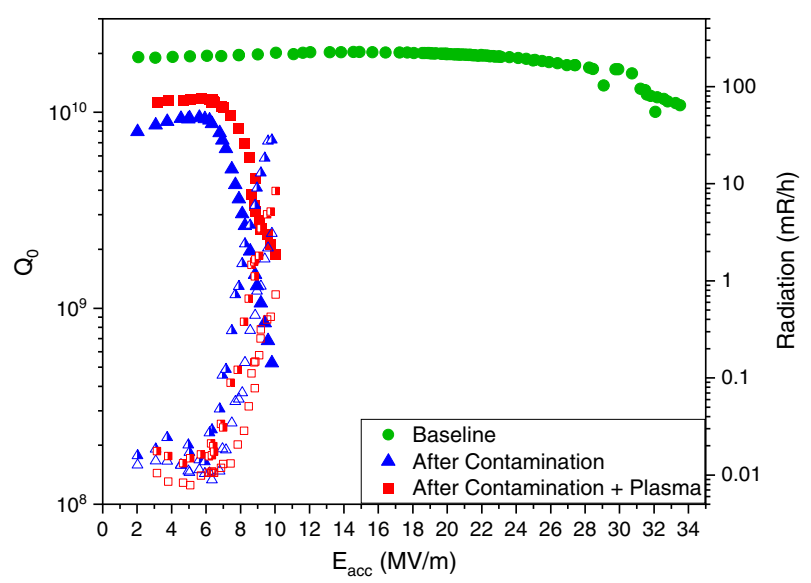

(b)

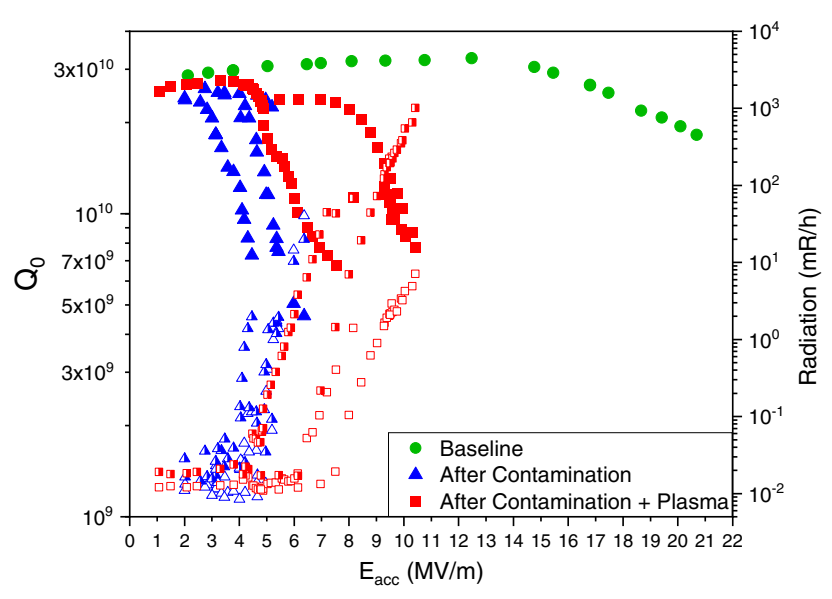

(d)

FIG. 10. Vacuum failure experiments simulated on four cavities. Part (a) shows the curves of the nine-cell cavity vented in the cleanroom; the results of the cold test performed after the cavity was contaminated are plotted in blue; the final radiation curves were acquired during this test at $1.4 \mathrm{~K}$ : they are plotted with a solid line and purple triangles (empty triangles for the top radiation detector, half-filled for the bottom detector). This cavity was plasma processed twice: the cold test conducted after the first round of plasma cleaning is plotted in red, and in black is the cold test performed after the second plasma round. Part (b) contains the rf tests of the singlecell cavity vented outside the cleanroom through the RAV (right angle valve); both rf tests (after the contamination and subsequently after the plasma test) were stopped at $10 \mathrm{MV} / \mathrm{m}$ before quenching; part (c) shows the results of the nine-cell cavity quickly vented through the mini RAV; plot (d) contains the curves of the nine-cell cavity slowly vented through the mini RAV. For comparison all the plots also contain the baseline curves of the cavities, measured before they were exposed to the vacuum failure simulations. Solid symbols are used to plot the $\mathrm{Q}_{0} \mathrm{vs} \mathrm{E}_{\mathrm{acc}}$ curve, with empty symbols for the radiation detected from the top detector, and half-filled symbols for the bottom radiation detector.

from the bottom to the top detector, and the respective onset was registered at 16 and $19 \mathrm{MV} / \mathrm{m}$ for bottom and top detector. This, along with the change in radiation intensity, suggests that some FE processing occurred during the rf test; however, the cavity continued to exhibit significant FE. The rf test measured after the first plasma processing is shown in red: the cavity quenched at $23 \mathrm{MV} / \mathrm{m}$ and no $\mathrm{x}$-ray activity was registered during the test, indicating that the FE was completely removed. The quality factor instead showed degradation: from $2.4 \times 10^{10}$ at $16.3 \mathrm{MV} / \mathrm{m}$ registered before plasma processing to $2.2 \times 10^{10}$ at $16.4 \mathrm{MV} / \mathrm{m}$ after plasma processing.
We applied a second round of plasma processing to the nine-cell cavity to investigate whether the quality factor degradation was caused by the plasma treatment or was due to a different amount of trapped flux, since the two rf tests (after the contamination and after the first plasma processing) were measured in different Dewars. After an additional $1 \mathrm{~h} 40$ min of plasma cleaning per cell, we cold tested the cavity. The curves are plotted in black in panel (a) of Fig. 10: the test confirms that the quality factor is actually preserved $\left(\mathrm{Q}_{0}=2.5 \times 10^{10}\right.$ at $\left.16.4 \mathrm{MV} / \mathrm{m}\right)$ and that both radiation detectors show no $\mathrm{x}$-ray activity, confirming that the FE was eliminated. 


\section{Vacuum failure experiments outside the cleanroom}

Following the experiment in the cleanroom, additional vacuum failures were simulated outside the cleanroom. The procedure was repeated with small variations in three cavities: one single-cell and two nine-cell cavities. After the contamination, the single-cell cavity was plasma processed for $20 \mathrm{~h}$, and the nine-cell cavities were processed for approximately $1 \mathrm{~h} 40 \mathrm{~min}$ per cell. After the venting, the cavities were evacuated to the low $10^{-6}$-high $10^{-7}$ Torr range; rf tests were performed before and after plasma processing. The plots in Fig. 10 show the $\mathrm{Q}_{0}$ and radiation versus $\mathrm{E}_{\mathrm{acc}}$ curves.

The single-cell cavity was quickly vented through the mini RAV from high vacuum to atmospheric pressure. Panel (b) in Fig. 10 presents the rf tests of the single-cell cavity. The green curve shows the baseline before the vacuum failure experiment (red curve in Fig. 9, $\mathrm{Q}_{0}=1.9 \times$ $10^{10}$ at $5 \mathrm{MV} / \mathrm{m}$ ). The contaminated cavity (blue curves) exhibits a degradation in quality factor $\left(\mathrm{Q}_{0}=9.4 \times 10^{9}\right.$ at $5 \mathrm{MV} / \mathrm{m}$ ) and radiation onset at $6.2 \mathrm{MV} / \mathrm{m}$. The test was limited by the available off power at $10 \mathrm{MV} / \mathrm{m}$, before quench. Due to the severity of FE registered during the first rf test it was decided to process the cavity for a longer duration than usual $(20 \mathrm{~h})$. The cold test conducted after plasma processing shows a moderate increase in quality factor $\left(\mathrm{Q}_{0}=1.2 \times 10^{10}\right.$ at $\left.5.1 \mathrm{MV} / \mathrm{m}\right)$ and decrease in radiation $(8.4 \mathrm{mR} / \mathrm{h}$ at $10 \mathrm{MV} / \mathrm{m}$ versus the $28 \mathrm{mR} / \mathrm{h}$ at $9.8 \mathrm{MV} / \mathrm{m}$ registered before plasma processing) with $\mathrm{FE}$ onset at $7 \mathrm{MV} / \mathrm{m}$.

Panel (c) in Fig. 10 shows the results of the nine-cell cavity quickly vented through the mini RAV. In green is plotted the baseline performance of the cavity before venting $\left(\mathrm{Q}_{0}=3.1 \times 10^{10}\right.$ at $\left.8.8 \mathrm{MV} / \mathrm{m}\right)$; in blue are the curves measured on the cavity after the contamination: quality factor degradation $\left(\mathrm{Q}_{0}=2.6 \times 10^{10}\right.$ at $\left.8.7 \mathrm{MV} / \mathrm{m}\right)$ and $\mathrm{FE}$ onset at $8.7 \mathrm{MV} / \mathrm{m}$, reach final quench at $17 \mathrm{MV} / \mathrm{m}$ with $1 \times 10^{4} \mathrm{mR} / \mathrm{h}$; the FE onset after quench was registered at $10.3 \mathrm{MV} / \mathrm{m}$. The curves after plasma treatment are shown in red: FE onset occurs at $8.8 \mathrm{MV} / \mathrm{m}$, with a less severe slope of the $\mathrm{Q}_{0}$ versus $\mathrm{E}_{\text {acc }}$ curve until $12.6 \mathrm{MV} / \mathrm{m}$; at this point an increase in radiation was registered along with a $\mathrm{Q}_{0}$ drop. The $\mathrm{Q}_{0}$ versus $\mathrm{E}_{\text {acc }}$ curve was measured, again showing overlap with the test measured on the contaminated cavity in terms of quench field $(17 \mathrm{MV} / \mathrm{m})$, quality factor degradation and slope due to FE.

We simulated the final vacuum failure experiment by slowly opening the mini RAV on a nine-cell cavity, venting the cavity over a $18 \mathrm{~min}$ time interval. The results of the rf tests are shown in panel (d) of Fig. 10. The green curve shows the baseline performance; in blue are plotted the curves of the contaminated cavity (before plasma processing): the cavity shows intense FE as evident from the slope in quality factor. A first quench was reached below $3 \mathrm{MV} / \mathrm{m}$, and we then increased the accelerating field:
FE started at $4 \mathrm{MV} / \mathrm{m}$ and there was a switch in quality factor (from $7.3 \times 10^{9}$ to $1.75 \times 10^{10}$ ) between 4.5-5 MV $/ \mathrm{m}$. The sudden jump in $\mathrm{Q}_{0}$ suggests that a field emitter was processed by the rf, eliminating the radiation and energy dissipation that it was causing. After this event, the quality factor followed a new curve that started bending above $4 \mathrm{MV} / \mathrm{m}$ while the radiation increased. Another $\mathrm{Q}_{0}$ switch occurred at $6.4 \mathrm{MV} / \mathrm{m}$, indicating that a new field emitter was likely processed. The test was stopped at this point due to intense FE $(>1 \mathrm{R} / \mathrm{h})$ and to avoid the risk of damaging the cavity surface through rf processing of field emitters; the final FE onset was registered at $5.2 \mathrm{MV} / \mathrm{m}$. In red is reported the rf test measured after plasma processing: the $\mathrm{Q}_{0}$ curve overlaps with the final curve before plasma processing (blue). Additionally, in this case a $\mathrm{Q}_{0}$ switch was observed at $7.5 \mathrm{MV} / \mathrm{m}$, indicating rf processing of the field emitter. The test was stopped at $10.4 \mathrm{MV} / \mathrm{m}$. The partial performance recovery observed in this cavity is to be attributed to rf processing which occurred during the $2 \mathrm{~K}$ vertical tests, not to plasma processing.

\section{CONCLUSIONS}

Using the newly developed technique of plasma ignition with HOMs, it was demonstrated that plasma processing successfully interacts with the cavity iris and removes C-based contamination.

It was also proved that plasma processing does not negatively affect the performance of nitrogen doped cavities: on the contrary, it preserves their high quality factor and quench field. Plasma cleaning was applied to multiple LCLS-II cavities with natural FE or artificially contaminated. The comparison between the rf tests conducted before and after plasma cleaning showed an increase in performance in the carbon contaminated single-cell, in one out of two naturally field emitting cavities and in one nine-cell cavity exposed to vacuum failure simulation inside the cleanroom. A second cavity with natural FE was processed, but still showed x-ray activity after the plasma, suggesting that the source of FE may not be C-related in this case, but due to metal flakes or surface defects. The three cavities used for vacuum failure simulation outside the cleanroom have shown little or no improvement attributable to plasma processing.

Plasma processing has achieved positive results in cavities with FE onset registered at high field levels (above $16 \mathrm{MV} / \mathrm{m}$ ), while it has not been able to reduce FE in cases in which the onset started at low fields. This can be related with the source of FE: plasma processing, applied with the current recipe and parameters, is effective in cases of hydrocarbon contamination, but not metal flakes, which represent the most plausible cause of FE at low fields.

To better understand what is the nature of the field emitters in the cavities exposed to vacuum failure simulations, it was decided to collect and analyze the particles introduced in the cavities and not removed by plasma 
cleaning. Preliminary results from the analysis of particles collected from the single-cell cavity suggest that metal flakes were introduced into the cavity during the vacuum failure experiment performed outside the cleanroom. The SEM/EDS analysis of the particles collected from the vented cavities is currently ongoing and will be the subject of future publication.

We intend to apply plasma processing to more LCLS-II nine-cell cavities and cold test them (before and after) in order to acquire additional statistics, focusing in particular on cavities that exhibit FE of unknown source (natural FE) during the rf tests.

\section{ACKNOWLEDGMENTS}

This work was supported by the U.S. Department of Energy (DOE), Office of Science, Basic Energy Sciences (BES). Fermilab is operated by Fermi Research Alliance, LLC under Contract No. DE-AC02-07CH11359 with the U.S. Department of Energy. We thank LCLS-II-HE for providing the $\mathrm{N}$-doped $1.3 \mathrm{GHz}$ nine-cell cavities used in this study for plasma processing and cold tests.

[1] J. Stohr, Linac coherent light source II (LCLS-II) conceptual design report, Technical Report, SLAC National Accelerator Laboratory, Menlo Park, CA, 2011.

[2] J. Galayda, The Linac Coherent Light Source-II Project, in Proceedings of the 5th International Particle Accelerator Conference (IPAC'14), Dresden, Germany, (JACoW, Geneva, Switzerland, 2014), pp. 935-937, https://accelconf.web.cern.ch/IPAC2014/papers/tuoca01 .pdf.

[3] A. Grassellino, A. Romanenko, D. Sergatskov, O. Melnychuk, Y. Trenikhina, A. Crawford, A. Rowe, M. Wong, T. Khabiboulline, and F. Barkov, Nitrogen and argon doping of niobium for superconducting radio frequency cavities: A pathway to highly efficient accelerating structures, Supercond. Sci. Technol. 26, 102001 (2013).

[4] A. Crawford, R. Eichhorn, F. Furuta, G. Ge, R. Geng, D. Gonnella, A. Grassellino, A. Hocker, G. Hoffstaetter, M. Liepe et al., The joint high $\mathrm{Q}_{0} \mathrm{R} \& \mathrm{D}$ program for LCLS-II, in Proceedings of the 5th International Particle Accelerator Conference (IPAC'14), Dresden, Germany (JACoW, Geneva, Switzerland, 2014), pp. 2627-2630, https:// accelconf.web.cern.ch/IPAC2014/papers/wepri062.pdf.

[5] A. Grassellino, A. Romanenko, S. Posen, Y. Trenikhina, O. Melnychuk, D. Sergatskov, M. Merio, M. Checchin, and M. Martinello, $\mathrm{N}$ doping: Progress in development and understanding, Proceedings of the 17th International Conference on RF Superconductivity (SRF'15), Whistler, BC, Canada (JACoW, Geneva, Switzerland, 2015), pp. 4854, https://accelconf.web.cern.ch/SRF2015/papers/ moba06.pdf.

[6] D. Gonnella, S. Aderhold, A. Burrill, E. Daly, K. Davis, A. Grassellino, C. Grimm, T. Khabiboulline, F. Marhauser, O. Melnychuk et al., Industrialization of the nitrogen-doping preparation for SRF cavities for LCLS-II, Nucl. Instrum. Methods Phys. Res., Sect. A 883, 143 (2018).

[7] B. W. McNeil and N. R. Thompson, X-ray free-electron lasers, Nat. Photonics 4, 814 (2010).

[8] C.-C. Kao, Challenges and opportunities for the next decade of XFELs, Nat. Rev. Phys. 2, 340 (2020).

[9] M. Cornacchia, Linac coherent light source (LCLS) design study report, Technical Report, Stanford Linear Accelerator Center, Menlo Park, CA, 1998.

[10] R. Schoenlein, S. Boutet, M. Minitti, and A. Dunne, The linac coherent light source: Recent developments and future plans, Appl. Sci. 7, 850 (2017).

[11] H. Padamsee, J. Knobloch, and T. Hays, RF Superconductivity for Accelerators (John Wiley \& Sons, New York, 1998).

[12] R. H. Fowler and L. Nordheim, Electron emission in intense electric fields, Proc. R. Soc. A 119, 173 (1928).

[13] P. S. Bagus, D. Käfer, G. Witte, and C. Wöll, Work Function Changes Induced by Charged Adsorbates: Origin of the Polarity Asymmetry, Phys. Rev. Lett. 100, 126101 (2008).

[14] M. Doleans, P. Tyagi, R. Afanador, C. McMahan, J. Ball, D. Barnhart, W. Blokland, M. Crofford, B. Degraff, S. Gold et al., In situ plasma processing to increase the accelerating gradients of superconducting radio-frequency cavities, Nucl. Instrum. Methods Phys. Res., Sect. A 812, 50 (2016).

[15] C. Cao, D. Ford, S. Bishnoi, T. Proslier, B. Albee, E. Hommerding, A. Korczakowski, L. Cooley, G. Ciovati, and J.F. Zasadzinski, Detection of surface carbon and hydrocarbons in hot spot regions of niobium SRF cavities by Raman spectroscopy, Phys. Rev. ST Accel. Beams 16, 064701 (2013).

[16] U. Pudasaini, G. V. Eremeev, C. E. Reece, J. Tuggle, and M. J. Kelley, Analysis of rf losses and material characterization of samples removed from a $\mathrm{Nb}_{3} \mathrm{Sn}$-coated superconducting rf cavity, Supercond. Sci. Technol. 33, 045012 (2020).

[17] T. Mason, D. Abernathy, I. Anderson, J. Ankner, T. Egami, G. Ehlers, A. Ekkebus, G. Granroth, M. Hagen, K. Herwig et al., The Spallation Neutron Source in Oak Ridge: A powerful tool for materials research, Physica (Amsterdam) 385B, 955 (2006).

[18] M. Doleans, Ignition and monitoring technique for plasma processing of multicell superconducting radio-frequency cavities, J. Appl. Phys. 120, 243301 (2016).

[19] M. Doleans, D. Vandygriff, S. Stewart, S. Gold, T. Neustadt, W. Strong, C. McMahan, S.-W. Lee, P. Tyagi, D. Vandygriff et al., Plasma processing to improve the performance of the SNS superconducting linac, in Proceedings of the 28th Linear Accelerator Conference (LINAC16), East Lansing, MI, US (JACoW, Geneva, Switzerland, 2016), pp. 679-682, https://accelconf.web .cern.ch/linac2016/papers/we2a03.pdf.

[20] B. Aune, R. Bandelmann, D. Bloess, B. Bonin, A. Bosotti, M. Champion, C. Crawford, G. Deppe, B. Dwersteg, D. Edwards et al., Superconducting TESLA cavities, Phys. Rev. ST Accel. Beams 3, 092001 (2000).

[21] P. Berrutti, M. Doleans, D. Gonnella, A. Grassellino, T. Khabiboulline, S. Kim, G. Lanza, M. Martinello, M. Ross, and K. Tippey, Update on plasma process- 
ing R\&D for LCLS-II, in Proceedings of the 9th International Particle Accelerator Conference (IPAC'18), Vancouver, BC, Canada (JACoW, Geneva, Switzerland, 2018), pp. 2656-2658, http://accelconf .web.cern.ch/ipac2018/papers/wepmk012.pdf.

[22] P. Berrutti, B. Giaccone, M. Martinello, A. Grassellino, T. Khabiboulline, M. Doleans, S. Kim, D. Gonnella, G. Lanza, and M. Ross, Plasma ignition and detection for in situ cleaning of $1.3 \mathrm{GHz}$ nine-cell cavities, J. Appl. Phys. 126, 023302 (2019).

[23] B. Giaccone, P. Berrutti, M. Doleans, D. Gonnella, A. Grassellino, G. Lanza, M. Martinello, M. Ross, and J. Zasadzinski, Plasma processing to reduce field emission in LCLS-II 1.3 GHz SRF cavities, in Proceedings of the 19th International Conference on RF Superconductivity (SRF'19), Dresden, Germany (JACoW Publishing, Geneva, Switzerland, 2019), pp. 1231-1238, http:// accelconf.web.cern.ch/srf2019/papers/frcab7.pdf.

[24] A. Wu, L. Yang, C. Hu, C. Li, S. Huang, Y. Li, Q. Chu, P. Xiong, H. Guo, W. Yue, and Y. Hu, In situ plasma cleaning to decrease the field emission effect of half-wave superconducting radio-frequency cavities, Nucl. Instrum. Methods Phys. Res., Sect. A 905, 61 (2018).

[25] A. Wu, S. Huang, C. Li, Q. Chu, H. Guo, P. Xiong, Y. Song, F. Pan, T. Tan, W. Yue, and S. Zhang, The cryostat results of carbon contamination and plasma cleaning for the field emission on the SRF cavity, in Proceedings of the 19th International Conference on RF Superconductivity (SRF'19), Dresden, Germany (JACOW Publishing, Geneva, Switzerland, 2019), pp. 1038-1040, http:// accelconf.web.cern.ch/srf2019/papers/thp064.pdf.

[26] S. Huang, Q. Chu, Y. He, C. Li, A. Wu, and S. Zhang, The effect of helium processing and plasma cleaning for low beta HWR cavity, in Proceedings of the 19th International Conference on RF Superconductivity (SRF'19), Dresden, Germany (JACoW Publishing, Geneva, Switzerland, 2019), pp. 1228-1230, https://accelconf.web.cern.ch/ srf2019/papers/frcab6.pdf.
[27] S. C. Brown et al., Introduction to Electrical Discharges in Gases (John Wiley \& Sons, New York, 1966).

[28] R. Fitzpatrick, Plasma Physics: An Introduction (CRC Press, Boca Raton, 2014).

[29] P. Tyagi, R. Afanador, M. Doleans, C. McMahan, and S.-H. Kim, Plasma processing of $\mathrm{Nb}$ surfaces for SRF cavities, in Proceedings of the 28th Linear Accelerator Conference (LINAC14), Geneva, Switzerland (JACoW, Geneva, Switzerland, 2014), pp. 323-325, https:// accelconf.web.cern.ch/linac2014/papers/mopp115.pdf.

[30] LCLS-II Final Design Report, LCLSII-1.1-DR-0251-R0.

[31] M. Doleans, R. Afanador, J. Ball, W. Blokland, M. Crofford, B. Degraff, D. Douglas, B. Hannah, M. Howell, S. Kim et al., Plasma processing R\&D for the SNS superconducting linac RF cavities, Proceedings of the 16th International Conference on RF Superconductivity (SRF'13), Paris, France (JACoW, Geneva, Switzerland, 2013), pp. 551-557, https://accelconf.web.cern.ch/ SRF2013/papers/tup057.pdf.

[32] J. Slater, Microwave electronics, Rev. Mod. Phys. 18, 441 (1946).

[33] O. Melnychuk, A. Grassellino, and A. Romanenko, Error analysis for intrinsic quality factor measurement in superconducting radio frequency resonators, Rev. Sci. Instrum. 85, 124705 (2014).

[34] F. Krueger and J. Larson, Chipmunk IV: Development of and experience with a new generation of radiation area monitors for accelerator applications, Nucl. Instrum. Methods Phys. Res., Sect. A 495, 20 (2002).

[35] M. Martinello, A. Grassellino, M. Checchin, A. Romanenko, O. Melnychuk, D. Sergatskov, S. Posen, and J. Zasadzinski, Effect of interstitial impurities on the field dependent microwave surface resistance of niobium, Appl. Phys. Lett. 109, 062601 (2016).

[36] Aquadag® E, Aqueous Deflocculated Acheson Graphite, Ladd Research, Williston, VT, USA. 